\title{
LIEB-THIRRING INEQUALITIES ON THE TORUS
}

\author{
ALEXEI ILYIN AND ARI LAPTEV
}

\begin{abstract}
We consider the Lieb-Thirring inequalities on the $d$-dimensional torus with arbitrary periods. In the space of functions with zero average with respect to the shortest coordinate we prove the LiebThirring inequalities for the $\gamma$-moments of the negative eigenvalues with constants independent of ratio of the periods. Applications to the attractors of the damped Navier-Stokes system are given.
\end{abstract}

\section{Introduction}

The Lieb-Thirring inequalities [27] give estimates for the $\gamma$-moments of the negative eigenvalues $-\nu_{j}$ of the Schrödinger operator

$$
-\Delta-V
$$

in $L_{2}\left(\mathbb{R}^{d}\right)$ :

$$
\sum_{\nu_{i} \leq 0}\left|\nu_{i}\right|^{\gamma} \leq \mathrm{L}_{\gamma, d} \int V(x)^{\gamma+d / 2} d x .
$$

Here $V \geq 0$ is a real valued potential sufficiently fast decaying at infinity.

Sharp values of the constants $\mathrm{L}_{\gamma, d}$ for all $\gamma \geq 3 / 2$ and all $d$ were found in [24]:

$$
\mathrm{L}_{\gamma, d}=\mathrm{L}_{\gamma, d}^{\mathrm{cl}}
$$

where

$$
\mathrm{L}_{\gamma, d}^{\mathrm{cl}}=\frac{1}{(2 \pi)^{d}} \int_{\mathbb{R}^{d}}\left(1-|\xi|^{2}\right)_{+}^{\gamma} d \xi=\frac{\Gamma(\gamma+1)}{2^{d} \pi^{d / 2} \Gamma(\gamma+d / 2+1)}
$$

is the semi-classical constant. For $1 \leq \gamma<3 / 2$ the best known bounds for $\mathrm{L}_{\gamma, d}$ were found in [10]:

$$
\mathrm{L}_{\gamma, d} \leq \frac{\pi}{\sqrt{3}} \mathrm{~L}_{\gamma, d}^{\mathrm{cl}}
$$

2010 Mathematics Subject Classification. 35P15, 26D10, 46E35, 35B41.

Key words and phrases. Lieb-Thirring inequalities, Schrödinger operators, interpolation inequalities, attractors, fractal dimension. 
Both in [24] and [10] (see also [4] and [15]) the key role is played by the corresponding one-dimensional Lieb-Thirring estimates for matrix-valued potentials.

If we consider the Lieb-Thirring inequalities on a compact manifold $M$, then we have to take care on the simple eigenvalue 0 of the Laplacian, so that instead of (1.1) we consider the Schrödinger operator

$$
-\Delta-\Pi(V \Pi \cdot)
$$

where $\Pi$ is the orthoprojection

$$
\Pi \varphi(x)=\varphi(x)-\frac{1}{|M|} \int_{M} \varphi(x) d M,
$$

and $|M|$ is the measure of $M$. Then estimate (1.2) still holds on $M$ with constant $\mathrm{L}_{\gamma, d}=\mathrm{L}_{\gamma, d}(M)$ depending on the geometric properties of $M$.

The spectral inequality (1.2) for 1-moments is equivalent to the inequality for orthonormal families. Let $\left\{\varphi_{j}\right\}_{j=1}^{N} \in H^{1}\left(\mathbb{R}^{d}\right)$ be an orthonormal family in $L_{2}\left(\mathbb{R}^{d}\right)$. (In the case of a manifold we have to assume that $\int_{M} \varphi_{j}(x) d M=0$.) Then $\rho_{\varphi}(x):=\sum_{j=1}^{N} \varphi_{j}(x)^{2}$ satisfies the inequality

$$
\int \rho_{\varphi}(x)^{1+2 / d} d x \leq \mathrm{k}_{d} \sum_{j=1}^{N}\left\|\nabla \varphi_{j}\right\|^{2},
$$

where the best constants $\mathrm{k}_{d}$ and $\mathrm{L}_{1, d}$ satisfy [27], [26]

$$
\mathrm{k}_{d}=(2 / d)(1+d / 2)^{1+2 / d} \mathrm{~L}_{1, d}^{2 / d} .
$$

In addition to the initial quantum mechanical applications inequality (1.5) is essential for finding good estimates for the dimension of the attractors in the theory of infinite dimensional dissipative dynamical systems, especially, for the attractors of the Navier-Stokes equations (see, for instance, [26], 2], [9], [12], [18], [31] and the references therein). Lieb-Thirring inequalities (1.5) were generalized to higher-order elliptic operators on domains with various boundary conditions and Riemannian manifolds [12], [31], however, with no information the corresponding constants. A different approach based on the methods of trigonometric series was proposed in [23].

For the two-dimensional square torus $\mathbb{T}^{2}$ an explicit bound for the LiebThirring constant $\mathrm{L}_{1,2}\left(\mathbb{T}^{2}\right)$ was obtained in [19]:

$$
\mathrm{L}_{1,2}\left(\mathbb{T}^{2}\right) \leq \frac{3}{8}
$$

Following the original approach in [27] the Birman-Schwinger kernel was used in [19], and the bound $3 / 8$ is the same as that for $\mathbb{R}^{2}$ in [27]. 
The Lieb-Thirring constants on the torus depend only on the ratio of the periods and on the elongated torus $\mathbb{T}_{\alpha}^{2}=(0,2 \pi / \alpha) \times(0,2 \pi)$ are unbounded as $\alpha \rightarrow 0$. This is due to the fact that the space $L_{2}\left(\mathbb{T}_{\alpha}^{2}\right)$ contains the subspace of (periodic) functions depending only on the long coordinate $x_{1}$, which gives that, for example, for the 1-moments we have

$$
\mathrm{L}_{1,2}\left(\mathbb{T}_{\alpha}^{2}\right)=\frac{\mathrm{L}_{1,2}\left(\mathbb{T}^{2}\right)}{\alpha}
$$

To see this we extend the functions in the direction $x_{2}$ by periodicity $1 / \alpha$ times (assuming that $1 / \alpha$ is integer) to reduce the treatment to the square torus, see 13 .

The orthogonal complement to the subspace $L_{2}(0,2 \pi / \alpha)$ of functions depending only on $x_{1}$ consists of functions $\varphi\left(x_{1}, x_{2}\right)$ that have zero average with respect to $x_{2}$ for all $x_{1}$ :

$$
\left\{\varphi(x), \int_{0}^{2 \pi} \varphi\left(x_{1}, t\right) d t=0, \forall x_{1} \in(0,2 \pi / \alpha)\right\} .
$$

Let $\mathrm{P}$ denote the corresponding orthoprojection. Then the Lieb-Thirring constants for the operator

$$
\mathcal{H}=-\Delta-\mathrm{P}(V(x) \mathrm{P} \cdot)
$$

on $\mathbb{T}_{\alpha}^{2}$ are independent of $\alpha$ (more precisely, have $\alpha$-independent upper bounds). This was first observed in [33], and explicit estimates were obtained in [18].

In this work we consider Lieb-Thirring inequalities for the operator (1.8) on the $d$-dimensional torus $\mathbb{T}_{\alpha}^{d}$

$$
\mathbb{T}_{\alpha}^{d}=\left(0, L_{1}\right) \times \cdots \times\left(0, L_{d-1}\right) \times(0,2 \pi),
$$

where $L_{i}=2 \pi / \alpha_{i}$ and the lengths of the periods are arranged in the nonincreasing order

$$
\alpha_{1} \leq \cdots \leq \alpha_{d-1} \leq \alpha_{d}=1
$$

Here $\mathrm{P}$ is the orthoprojection

$$
(\mathrm{P} \psi)\left(x_{1}, \ldots, x_{d}\right)=\psi\left(x_{1}, \ldots, x_{d}\right)-\frac{1}{2 \pi} \int_{0}^{2 \pi} \psi\left(x_{1}, \ldots, x_{d-1}, t\right) d t,
$$

so that the resulting function has zero average with respect to shortest coordinate $x_{d}$ for all $x_{1}, \ldots, x_{d-1}$.

We can now state our main result. 
Theorem 1.1. Let $d \leq 19$. Then for any $\alpha$ satisfying (1.10) the negative eigenvalues of the operator (1.8) on the torus $\mathbb{T}_{\alpha}^{d}$ satisfy for $\gamma \geq 1$ the bound (1.2) with

$$
\mathrm{L}_{\gamma, d} \leq\left(\frac{\pi}{\sqrt{3}}\right)^{d} \mathrm{~L}_{\gamma, d}^{\mathrm{cl}}
$$

where $\mathrm{L}_{\gamma, d}^{\mathrm{cl}}$ is the semi-classical constant (1.3).

The main idea is to write the Laplacian in (1.8) in the form

$$
-\Delta=\left(-\frac{d^{2}}{d x_{1}^{2}}+\alpha_{1}^{2} \beta_{1}\right)+\cdots+\left(-\frac{d^{2}}{d x_{d-1}^{2}}+\alpha_{d-1}^{2} \beta_{d-1}\right)+\left(-\frac{d^{2}}{d x_{d}^{2}}-\delta\right),
$$

where

$$
\delta=\alpha_{1}^{2} \beta_{1}+\cdots+\alpha_{d-1}^{2} \beta_{d-1},
$$

and where $\beta_{j}>0$ are chosen so that $\delta<1$.

For $j=1, \ldots, d-1$ each operator

$$
-\frac{d^{2}}{d x_{j}^{2}}+\alpha^{2} \beta_{j}, \quad j=1, \ldots, d-1
$$

is invertible on $L_{2}\left(0,2 \pi / \alpha_{j}\right)_{\text {per }}$, while for $\delta<1$ the operator

$$
-\frac{d^{2}}{d x_{d}^{2}}-\delta
$$

is invertible on $\dot{L}_{2}(0,2 \pi)=L_{2}(0,2 \pi) \cap\left\{\psi: \int_{0}^{2 \pi} \psi(t) d t=0\right\}$.

Accordingly, in Section 2, we consider for these two types of operators the interpolation inequalities of $L_{\infty}-L_{2}-L_{2}$ type:

$$
\|u\|_{\infty}^{2} \leq K_{1}(\beta)\left(\int_{0}^{2 \pi / \alpha}\left(u^{\prime}(x)^{2}+\alpha^{2} \beta u(x)^{2}\right) d x\right)^{1 / 2}\left(\int_{0}^{2 \pi / \alpha} u(x)^{2} d x\right)^{1 / 2}
$$

where $\beta>0$ and $u \in H^{1}(0,2 \pi / \alpha)_{\text {per }}$; and

$$
\|u\|_{\infty}^{2} \leq K_{2}(\beta)\left(\int_{0}^{2 \pi}\left(u^{\prime}(x)^{2}-\beta u(x)^{2}\right) d x\right)^{1 / 2}\left(\int_{0}^{2 \pi} u(x)^{2} d x\right)^{1 / 2},
$$

where $\beta<1$ and $u \in \dot{H}^{1}(0,2 \pi / \alpha)_{\text {per }}=H^{1}(0,2 \pi / \alpha) \cap \int_{0}^{2 \pi} u(x) d x=0$. We find sharp constants in these inequalities and, in particular, show that $K_{1}(\beta)=1$ for $\beta \geq \beta_{*}=0.045 \ldots$ and $K_{2}(\beta)=1$ for $\beta \leq \beta_{* *}=0.839 \ldots$

In Section 3 we use the information on the sharp constants $K_{1}(\beta)$ and $K_{2}(\beta)$ to obtain following [20, Theorem 6.1] one dimensional inequalities of the type (1.5) for traces of matrices built from orthonormal families of 
vector functions. Then using the equivalence (1.6) we recast these results into the Lieb-Thirring inequalities for the negative eigenvalues $\left\{-\lambda_{j}\right\}$ and $\left\{-\mu_{j}\right\}$ of the one dimensional operators with matrix-valued potentials

$$
\begin{aligned}
& H_{1}(\beta)=-\frac{d^{2}}{d x^{2}}+\alpha^{2} \beta-V(x), \quad \beta>0, \quad x \in(0,2 \pi / \alpha), \\
& H_{2}(\delta)=-\frac{d^{2}}{d x^{2}}-\delta-\mathrm{P}(V(x) \mathrm{P} \cdot), \quad \delta \in[0,1), \quad x \in(0,2 \pi),
\end{aligned}
$$

acting on $L_{2}(0,2 \pi / \alpha)$ and $\dot{L}_{2}(0,2 \pi)=\left\{f \in L_{2}(0,2 \pi), \int_{0}^{2 \pi} f(x) d x=0\right\}$, respectively.

For a non-negative $M \times M$ Hermitian matrix $V$ we obtain

$$
\begin{aligned}
& \sum_{j} \lambda_{j} \leq \frac{2}{3 \sqrt{3}} K_{1}(\beta) \int_{0}^{2 \pi / \alpha} \operatorname{Tr}\left[V(x)^{3 / 2}\right] d x, \\
& \sum_{j} \mu_{j} \leq \frac{2}{3 \sqrt{3}} K_{2}(\delta) \int_{0}^{2 \pi} \operatorname{Tr}\left[V(x)^{3 / 2}\right] d x .
\end{aligned}
$$

Then we use the Aizenmann-Lieb argument [1] to obtain the estimates for the Riesz means of order $\gamma \geq 1$.

In Section 4 we use the lifting argument with respect to dimensions [24] and the one-dimensional Lieb-Thirring inequalities for matrix-valued potentials from Section 3 to prove Theorem 4.1 (to which Theorem 1.1 is a corollary). We point out here that the condition $d \leq 19$ (which is $d \leq\left[\beta_{* *} / \beta_{*}\right]+1$ ) makes it possible to chose $\beta_{j} \geq \beta_{*}$ and $\delta \leq \beta_{* *}$ so that in (1.16), (1.17) we have $K_{1}\left(\beta_{j}\right)=1$ and $K_{2}(\delta)=1$.

Finally, in Section 4 we give applications to the attractors of the dampeddriven Navier-Stokes equations on the square and elongated torus and improve the estimates of the dimension of the attractor obtained earlier in [21] and [22].

\section{TWO INTERPOLATION INEQUALITIES}

First inequality. Let $x \in[0,2 \pi / \alpha]_{\text {per }}$ and let $\alpha>0$. We consider the interpolation inequality (1.12) where $u \in H^{1}(0,2 \pi / \alpha)_{\text {per }}, \beta>0$ (and no orthogonality to constants is assumed). More precisely, we are interested in the value of the sharp constant $K_{1}(\beta)$ in this inequality.

The system

$$
\left\{\sqrt{\frac{\alpha}{2 \pi}} e^{i k \alpha x}\right\}_{k \in \mathbb{Z}}
$$


is a complete orthonormal system of eigenfunctions of the operator $-\frac{d^{2}}{d x^{2}}$ with periodic boundary conditions. Therefore the Green's function $G_{\lambda}(x, \xi)$, that is, the solution of the equation

$$
\mathbb{A}(\lambda) G_{\lambda}(x, \xi)=\delta(x-\xi),
$$

where

$$
\mathbb{A}(\lambda)=-\frac{d^{2}}{d x^{2}}+\alpha^{2} \beta+\lambda, \quad \lambda>0,
$$

is given by the series

$$
G_{\lambda}(x, \xi)=\frac{\alpha}{2 \pi} \sum_{k \in \mathbb{Z}} \frac{e^{i k \alpha(x-\xi)}}{\alpha^{2} k^{2}+\alpha^{2} \beta+\lambda} .
$$

On the diagonal

$$
G_{\lambda}(\xi, \xi)=\frac{\alpha}{2 \pi} \sum_{k \in \mathbb{Z}} \frac{1}{\alpha^{2} k^{2}+\alpha^{2} \beta+\lambda}=: g_{\beta}(\lambda) .
$$

Here we omit the subscript $\alpha$ on the right because $\alpha$ is fixed and, secondly, the sharp constant $K_{1}(\beta)$ is independent of $\alpha$, as our final result shows. Using the general result (Theorem 2.2 in 32 with $\theta=1 / 2$ ) we have the following expression for the sharp constant $K_{1}(\beta)$ :

$$
K_{1}(\beta)=\frac{1}{\theta^{\theta}(1-\theta)^{1-\theta}} \cdot \sup _{\lambda>0} \lambda^{\theta} g_{\beta}(\lambda)=2 \sup _{\lambda>0} \sqrt{\lambda} g_{\beta}(\lambda)
$$

(We point out that for the proof of (2.2) we can use the direct argument similar to the one used in Remark 3.1 in [20.) Next, using the formula

$$
\sum_{k \in \mathbb{Z}} \frac{1}{k^{2}+\lambda}=\frac{\pi \operatorname{coth}(\pi \sqrt{\lambda})}{\sqrt{\lambda}}
$$

in (2.1) we find that

$$
g_{\beta}(\lambda)=\frac{1}{2 \alpha} \frac{\operatorname{coth}\left(\pi \sqrt{\beta+\lambda / \alpha^{2}}\right)}{\sqrt{\beta+\lambda / \alpha^{2}}} .
$$

Since $\alpha>0$ is fixed, we can replace the variable $\lambda$ in (2.2) by $\alpha^{2} \lambda$, which finally gives

$$
K_{1}(\beta)=\sup _{\lambda>0} \sqrt{\lambda} \frac{\operatorname{coth}(\pi \sqrt{\beta+\lambda})}{\sqrt{\beta+\lambda}} .
$$

Since for every fixed $\beta>0$

$$
\lim _{\lambda \rightarrow \infty} \sqrt{\lambda} \frac{\operatorname{coth}(\pi \sqrt{\beta+\lambda})}{\sqrt{\beta+\lambda}}=1,
$$


it follows that

$$
K_{1}(\beta) \geq 1 \text {. }
$$

Next, (2.1) and (2.2) show that $K_{1}(\beta)$ is monotone non-increasing

$$
\beta_{1}<\beta_{2} \Rightarrow g_{\beta_{1}}(\lambda)>g_{\beta_{2}}(\lambda) \quad \Rightarrow \quad K_{1}\left(\beta_{1}\right) \geq K_{1}\left(\beta_{2}\right),
$$

and, finally,

$$
K_{1}\left(\beta_{0}\right)=1 \Rightarrow K_{1}(\beta)=1 \text { for } \beta \geq \beta_{0} .
$$

The graphs of the function $\lambda \rightarrow \sqrt{\lambda} \frac{\operatorname{coth}(\pi \sqrt{\beta+\lambda})}{\sqrt{\beta+\lambda}}$ are shown in Fig. 11. For very small $\beta$ the graphs have a sharp peak near the origin. For $\beta_{*}=0.045 \ldots$ (found numerically) the supremum is equal to 1 . Hence $K_{1}(\beta)=1$ for all $\beta \geq \beta_{*}$.

Thus, we have proved the following theorem.

Theorem 2.1. The sharp constant $K_{1}(\beta)$ in inequality (1.12) is given by (2.4). Furthermore, for all $\beta \geq \beta_{*}=0.045 \ldots, K_{1}(\beta)=1$.
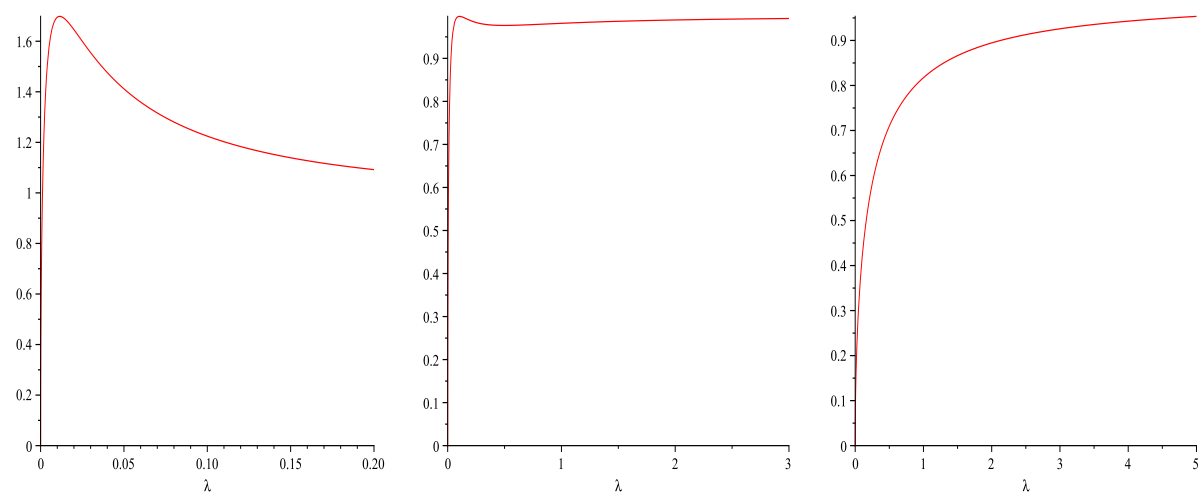

FigurE 1. Graphs of $\lambda \rightarrow \sqrt{\lambda} \frac{\operatorname{coth}(\pi \sqrt{\beta+\lambda})}{\sqrt{\beta+\lambda}}$ for $\beta=0.01, \beta_{*}, 0.5$.

Second inequality. Now let $x \in[0,2 \pi]$, and we consider inequality (1.13), where $u \in \dot{H}^{1}(0,2 \pi)_{\text {per }}=H^{1}(0,2 \pi)_{\text {per }} \cap \int_{0}^{2 \pi} u(x) d x=0$, and $0 \leq \beta<1$.

The Green's function $G_{\lambda}(x, \xi)$ of the operator

$$
\mathbb{A}(\lambda)=-\frac{d^{2}}{d x^{2}}-\beta+\lambda, \quad \lambda>0,
$$

in the space of functions with mean value zero is as follows

$$
G_{\lambda}(x, \xi)=\frac{1}{2 \pi} \sum_{k \in \mathbb{Z}_{0}} \frac{e^{i k(x-\xi)}}{k^{2}-\beta+\lambda}, \quad \mathbb{Z}_{0}=\mathbb{Z} \backslash\{0\},
$$


and

$$
G_{\lambda}(\xi, \xi)=\frac{1}{2 \pi} \sum_{k \in \mathbb{Z}_{0}} \frac{1}{k^{2}-\beta+\lambda}=: f_{\beta}(\lambda) .
$$

As before we have the following expression for the sharp constant $K_{2}(\beta)$ :

$$
K_{2}(\beta)=2 \sup _{\lambda>0} \sqrt{\lambda} f_{\beta}(\lambda) .
$$

Next, from (2.3) we obviously have

$$
\sum_{k \in \mathbb{Z}_{0}} \frac{1}{k^{2}+\lambda}=\frac{\pi \sqrt{\lambda} \operatorname{coth}(\pi \sqrt{\lambda})-1}{\lambda},
$$

giving

$$
f_{\beta}(\lambda)=\frac{1}{2} \frac{\sqrt{\lambda-\beta} \operatorname{coth}(\pi \sqrt{\lambda-\beta})-1 / \pi}{\lambda-\beta}
$$

and, finally,

$$
K_{2}(\beta)=\sup _{\lambda>0} \sqrt{\lambda} \frac{\sqrt{\lambda-\beta} \operatorname{coth}(\pi \sqrt{\lambda-\beta})-1 / \pi}{\lambda-\beta} .
$$

The existence of the limit

$$
\lim _{\lambda \rightarrow \infty} \sqrt{\lambda} \frac{\sqrt{\lambda-\beta} \operatorname{coth}(\pi \sqrt{\lambda-\beta})-1 / \pi}{\lambda-\beta}=1,
$$

implies that

$$
K_{2}(\beta) \geq 1
$$

This time $K_{2}(\beta)$ is monotone non-decreasing

$$
\beta_{1}<\beta_{2} \Rightarrow f_{\beta_{1}}(\lambda)<f_{\beta_{2}}(\lambda) \quad \Rightarrow \quad K_{1}\left(\beta_{1}\right) \leq K_{1}\left(\beta_{2}\right),
$$

and, finally,

$$
K_{2}\left(\beta_{0}\right)=1,0<\beta_{0}<1 \Rightarrow K_{2}(\beta)=1 \quad \text { for } \quad 0 \leq \beta \leq \beta_{0} .
$$

The graphs of the function $\lambda \rightarrow \sqrt{\lambda} \frac{\sqrt{\lambda-\beta} \operatorname{coth}(\pi \sqrt{\lambda-\beta})-1 / \pi}{\lambda-\beta}$ are shown in Fig. 2, which is somewhat symmetrical to Fig. 11. For $\beta$ close to 1 the graphs have a sharp peak near the origin. For $\beta_{* *}=0.839 \ldots$ (again found numerically) the supremum is equal to 1 and is attained both at a finite $\lambda$ and at infinity. Hence $K_{1}(\beta)=1$ for all $\beta \geq \beta_{* *}$.

Thus, we have proved the following theorem.

Theorem 2.2. The sharp constant $K_{2}(\beta)$ in inequality (1.13) is given by (2.6). Furthermore, for all $\beta \leq \beta_{* *}=0.839 \ldots, K_{2}(\beta)=1$. 
Remark 2.1. Inequality (1.13) with $\beta=0$ and $K_{2}(0)=1$ goes back to [14] where it was used for the proof of the Carlson inequality. Sharp constants in the higher-order inequalities were found in [30] for $x \in \mathbb{R}$ and in [17] for $x \in \mathbb{S}^{1}$. Various refinements and improvements of this type of inequalities were recently obtained in [3], [20], 32].
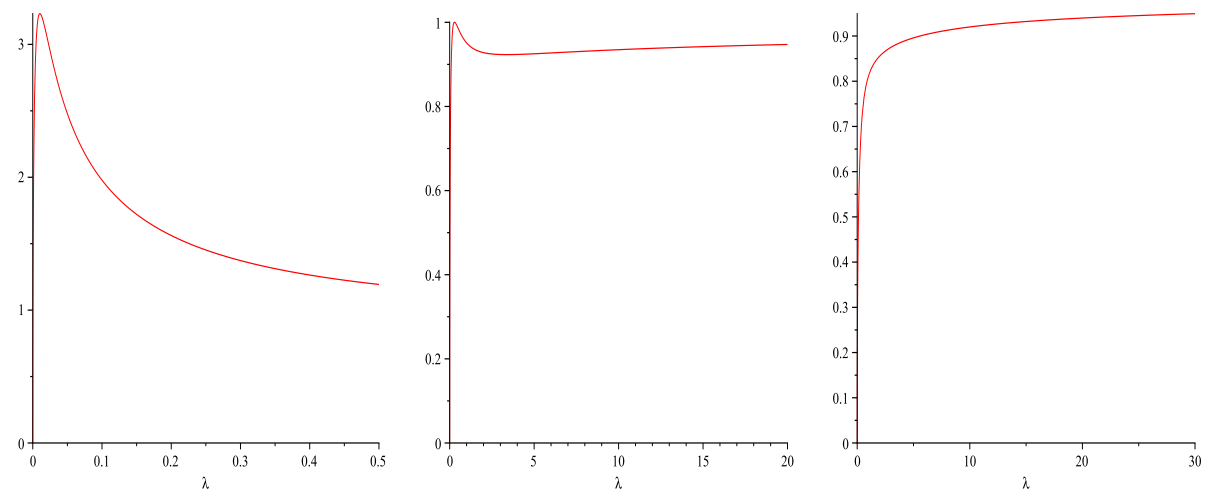

Figure 2. Graphs of $\lambda \rightarrow \sqrt{\lambda} \frac{\sqrt{\lambda-\beta} \operatorname{coth}(\pi \sqrt{\lambda-\beta})-1 / \pi}{\lambda-\beta}$ for $\beta=0.99, \beta_{* *}, 0.5$.

Remark 2.2. By the definition of $K_{1}(\beta)$ and $K_{2}(\beta)$ we have

$$
\begin{aligned}
& \sum_{k \in \mathbb{Z}} \frac{1}{k^{2}+\beta+\lambda} \leq K_{1}(\beta) \frac{\pi}{\sqrt{\lambda}}, \quad \beta>0, \\
& \sum_{k \in \mathbb{Z}_{0}} \frac{1}{k^{2}-\beta+\lambda} \leq K_{2}(\beta) \frac{\pi}{\sqrt{\lambda}}, \quad \beta \in[0,1) .
\end{aligned}
$$

\section{One-Dimensional Sobolev inequalities for traces of} MATRICES AND ASSOCIATED LIEB-THIRRING INEQUALITIES

Let $\left\{\phi_{n}\right\}_{n=1}^{N}$ be an orthonormal family of periodic vector-functions

$$
\phi_{n}(x)=\left(\phi_{n}(x, 1), \ldots, \phi_{n}(x, M)\right)^{T}
$$

defined for $x \in[0,2 \pi / \alpha]_{\text {per }}$ :

$$
\left(\phi_{n}, \phi_{m}\right)=\sum_{j=1}^{M} \int_{0}^{L} \phi_{n}(x, j) \overline{\phi_{m}(x, j)} d x=\int_{0}^{L} \phi_{n}(x)^{T} \overline{\phi_{m}(x)} d x=\delta_{n m},
$$

where we set for brevity

$$
L:=2 \pi / \alpha .
$$


We consider the $M \times M$ matrix $U(x, y)$

$$
U(x, y)=\sum_{n=1}^{N} \phi_{n}(x){\overline{\phi_{n}(y)}}^{T}
$$

with entries $[U(x, y)]_{j k}=\sum_{n=1}^{N} \phi_{n}(x, j) \overline{\phi_{n}(y, k)}$. Clearly,

$$
U(x, y)^{*}=U(y, x)
$$

and by orthonormality

$$
\begin{array}{r}
\int_{0}^{L} U(x, y) U(y, z) d y=\sum_{n, n^{\prime}=1}^{N} \int_{0}^{L} \phi_{n}(x){\overline{\phi_{n}(y)}}^{T} \phi_{n^{\prime}}(y){\overline{\phi_{n^{\prime}}(z)}}^{T} d y= \\
=\sum_{n=1}^{N} \phi_{n}(x){\overline{\phi_{n}(z)}}^{T}=U(x, z) .
\end{array}
$$

Theorem 3.1. Let $\phi_{n}(x, j) \in H^{1}(0, L)_{\mathrm{per}}$. Then

$$
\int_{0}^{L} \operatorname{Tr}\left[U(x, x)^{3}\right] d x \leq K_{1}(\beta)^{2} \sum_{n=1}^{N} \sum_{j=1}^{M} \int_{0}^{L}\left(\left|\phi_{n}^{\prime}(x, j)\right|^{2}+\alpha^{2} \beta\left|\phi_{n}(x, j)\right|^{2}\right) d x,
$$

where $K_{1}(\beta)$ is defined in (1.12) and $\beta>0$.

$$
\begin{aligned}
& \text { If } \alpha=1, L=2 \pi \text {, and } \int_{0}^{2 \pi} \phi_{n}(x, j)=0 \text { for all } n \text { and } j \text {, then } \\
& \int_{0}^{2 \pi} \operatorname{Tr}\left[U(x, x)^{3}\right] d x \leq K_{2}(\beta)^{2} \sum_{n=1}^{N} \sum_{j=1}^{M} \int_{0}^{2 \pi}\left(\left|\phi_{n}^{\prime}(x, j)\right|^{2}-\beta\left|\phi_{n}(x, j)\right|^{2}\right) d x,
\end{aligned}
$$

where $K_{2}(\beta)$ is defined in (1.13) and $\beta \in[0,1)$.

Proof. Both inequalities are proved similarly. The argument, in turn, follows very closely the proof of Theorem 6.1 in [20]. Let us prove, for example, the second inequality (3.3). We have

$$
\tilde{U}(n, x)=\frac{1}{\sqrt{2 \pi}} \int_{0}^{2 \pi} e^{-i y n} U(y, x) d y, \quad n \in \mathbb{Z}_{0},
$$

so that

$$
U(y, x)=\frac{1}{\sqrt{2 \pi}} \sum_{k \in \mathbb{Z}_{0}} e^{i y k} \tilde{U}(k, x)
$$


Next,

$$
\sum_{k \in \mathbb{Z}_{0}} \tilde{U}(k, x)^{*} \tilde{U}(k, x)=\int_{0}^{2 \pi} U(y, x)^{*} U(y, x) d y=U(x, x),
$$

and

$$
\begin{array}{r}
\sum_{k \in \mathbb{Z}_{0}}\left(k^{2}-\beta\right) \tilde{U}(k, x)^{*} \tilde{U}(k, x)=\int_{0}^{2 \pi}\left[\partial_{y} U(y, x)\right]^{*} \partial_{y} U(y, x) d y- \\
\quad-\beta \int_{0}^{L} U(y, x)^{*} U(y, x) d y,
\end{array}
$$

where the last term is equal to $-\beta U(x, x)$. Now, by orthonormality

$$
\begin{aligned}
& \operatorname{Tr}\left[\int_{0}^{2 \pi} \sum_{k \in \mathbb{Z}_{0}}\left(k^{2}-\beta\right) \tilde{U}(k, x)^{*} \tilde{U}(k, x) d x\right]= \\
& \operatorname{Tr}\left[\int_{0}^{2 \pi} \int_{0}^{2 \pi} \sum_{n, n^{\prime}=1}^{N} \phi_{n^{\prime}}^{\prime}(y){\overline{\phi_{n^{\prime}}(x)}}^{T} \phi_{n}(x){\overline{\phi_{n}^{\prime}(y)}}^{T} d x d y-\beta \int_{0}^{2 \pi} U(x, x) d x\right]= \\
& \operatorname{Tr}\left[\int_{0}^{2 \pi} \sum_{n=1}^{N}\left[\phi_{n}^{\prime}(y){\overline{\phi_{n}^{\prime}(y)}}^{T}-\beta \phi_{n}(y){\overline{\phi_{n}(y)}}^{T}\right] d y\right]= \\
& \quad=\sum_{n=1}^{N} \sum_{j=1}^{M} \int_{0}^{2 \pi}\left(\left|\phi_{n}^{\prime}(x, j)\right|^{2}-\beta\left|\phi_{n}(x, j)\right|^{2}\right) d x .
\end{aligned}
$$

Now consider

$$
\begin{aligned}
& \operatorname{Tr}\left[U(x, x)^{3}\right]=\sum_{k \in \mathbb{Z}_{0}} \operatorname{Tr}\left[U(x, x)^{2} \tilde{U}(k, x)\right] \frac{e^{i x k}}{\sqrt{2 \pi}}= \\
& \sum_{k \in \mathbb{Z}_{0}} \operatorname{Tr}\left[\left[\left(k^{2}-\beta\right) I+\Lambda(x)^{2}\right]^{-1 / 2} U(x, x)^{2} \tilde{U}(k, x)\left[\left(k^{2}-\beta\right) I+\Lambda(x)^{2}\right]^{1 / 2}\right] \frac{e^{i x k}}{\sqrt{2 \pi}},
\end{aligned}
$$

where $\Lambda(x)$ is an arbitrary positive definite matrix. Using below the CauchySchwartz inequality for matrices we get the upper bounds

$$
\begin{aligned}
& \sqrt{2 \pi} \operatorname{Tr}\left[U(x, x)^{3}\right] \leq \\
& \sum_{k \in \mathbb{Z}_{0}}\left|\operatorname{Tr}\left[\left[\left(k^{2}-\beta\right) I+\Lambda(x)^{2}\right]^{-1 / 2} U(x, x)^{2} \tilde{U}(k, x)\left[\left(k^{2}-\beta\right) I+\Lambda(x)^{2}\right]^{1 / 2}\right]\right| \leq \\
& \sum_{k \in \mathbb{Z}_{0}}\left(\operatorname{Tr}\left[U(x, x)^{2}\left[\left(k^{2}-\beta\right) I+\Lambda(x)^{2}\right]^{-1} U(x, x)^{2}\right]\right)^{1 / 2} \times
\end{aligned}
$$




$$
\begin{aligned}
& \left(\operatorname{Tr}\left[\left[\left(k^{2}-\beta\right) I+\Lambda(x)^{2}\right] \tilde{U}(k, x)^{*} \tilde{U}(k, x)\right]\right)^{1 / 2} \leq \\
& \left(\sum_{k \in \mathbb{Z}_{0}} \operatorname{Tr}\left[U(x, x)^{2}\left[\left(k^{2}-\beta\right) I+\Lambda(x)^{2}\right]^{-1} U(x, x)^{2}\right]\right)^{1 / 2} \times \\
& \left(\sum_{k \in \mathbb{Z}_{0}} \operatorname{Tr}\left[\left[\left(k^{2}-\beta\right) I+\Lambda(x)^{2}\right] \tilde{U}(k, x)^{*} \tilde{U}(k, x)\right]\right)^{1 / 2} .
\end{aligned}
$$

We now use the matrix inequality

$$
\sum_{k \in \mathbb{Z}_{0}}\left[\left(k^{2}-\beta\right) I+\Lambda(x)^{2}\right]^{-1}<K_{2}(\beta) \pi \Lambda(x)^{-1},
$$

which follows from the scalar inequality (see (2.7))

$$
\sum_{k \in \mathbb{Z}_{0}} \frac{1}{k^{2}-\beta+\lambda^{2}}<K_{2}(\beta) \pi \lambda^{-1}
$$

applied to each eigenvector $e=e(x)$ of $\Lambda(x)$ with eigenvalue $\lambda=\lambda(x)>0$ from the orthonormal basis $\left\{e_{j}(x), \lambda_{j}(x)\right\}_{j=1}^{M}$. This gives for the first factor

$$
\begin{array}{r}
\sum_{k \in \mathbb{Z}_{0}} \operatorname{Tr}\left[U(x, x)^{2}\left[\left(k^{2}-\beta\right) I+\Lambda(x)^{2}\right]^{-1} U(x, x)^{2}\right]= \\
\operatorname{Tr}\left[U(x, x)^{2} \sum_{k \in \mathbb{Z}_{0}}\left[\left(k^{2}-\beta\right) I+\Lambda(x)^{2}\right]^{-1} U(x, x)^{2}\right] \leq \\
K_{2}(\beta) \pi \operatorname{Tr}\left[U(x, x)^{2} \Lambda(x)^{-1} U(x, x)^{2}\right] .
\end{array}
$$

For the second factor we simply have

$$
\begin{array}{r}
\sum_{k \in \mathbb{Z}_{0}} \operatorname{Tr}\left[\left[\left(k^{2}-\beta\right) I+\Lambda(x)^{2}\right] \tilde{U}(k, x)^{*} \tilde{U}(k, x)\right]= \\
\sum_{k \in \mathbb{Z}_{0}} \operatorname{Tr}\left[\left(k^{2}-\beta\right) \tilde{U}(k, x)^{*} \tilde{U}(k, x)\right]+\sum_{k \in \mathbb{Z}_{0}} \operatorname{Tr}\left[\Lambda(x)^{2} \tilde{U}(k, x)^{*} \tilde{U}(k, x)\right]
\end{array}
$$

If we now chose $\Lambda(x)=\gamma(U(x, x)+\varepsilon I), \gamma>0$, and let $\varepsilon \rightarrow 0$ we obtain (observing that $\lambda^{4} /(\lambda+\varepsilon) \rightarrow \lambda^{3}$ as $\varepsilon \rightarrow 0$ for $\lambda \geq 0$; this is required in case when $U(x, x)$ is not invertible)

$$
\begin{gathered}
\sqrt{2 \pi} \operatorname{Tr}\left[U(x, x)^{3}\right] \leq \sqrt{\pi K_{2}(\beta)} \gamma^{-1 / 2} \operatorname{Tr}\left[U(x, x)^{3}\right]^{1 / 2} \times \\
\left(\sum_{k \in \mathbb{Z}_{0}} \operatorname{Tr}\left[\left(k^{2}-\beta\right) \tilde{U}(k, x)^{*} \tilde{U}(k, x)\right]+\gamma^{2} \operatorname{Tr}\left[U(x, x)^{3}\right]\right),
\end{gathered}
$$


where we have also used (3.4), or

$$
\begin{gathered}
\operatorname{Tr}\left[U(x, x)^{3}\right] \leq \\
\frac{K_{2}(\beta)}{2}\left(\gamma^{-1} \sum_{k \in \mathbb{Z}_{0}} \operatorname{Tr}\left[\left(k^{2}-\beta\right) \tilde{U}(k, x)^{*} \tilde{U}(k, x)\right]+\gamma \operatorname{Tr}\left[U(x, x)^{3}\right]\right) .
\end{gathered}
$$

If we optimize over $\gamma$, we obtain

$$
\operatorname{Tr}\left[U(x, x)^{3}\right] \leq K_{2}(\beta)\left(\operatorname{Tr}\left[U(x, x)^{3}\right]\right)^{1 / 2}\left(\sum_{k \in \mathbb{Z}_{0}} \operatorname{Tr}\left[\left(k^{2}-\beta\right) \tilde{U}(k, x)^{*} \tilde{U}(k, x)\right]\right)^{1 / 2}
$$

or

$$
\operatorname{Tr}\left[U(x, x)^{3}\right] \leq K_{2}(\beta)^{2} \sum_{k \in \mathbb{Z}_{0}} \operatorname{Tr}\left[\left(k^{2}-\beta\right) \tilde{U}(k, x)^{*} \tilde{U}(k, x)\right] .
$$

If we integrate with respect to $x$ and use (3.5), we obtain (3.3). The proof of the theorem is complete since the proof of (3.2) is totally similar.

Remark 3.1. In the scalar case $M=1$ inequalities (3.2), (3.3) go over to

$$
\begin{aligned}
& \int_{0}^{L}\left(\sum_{n=1}^{N}\left|\phi_{n}(x)\right|^{2}\right)^{3} d x \leq K_{1}(\beta)^{2} \sum_{n=1}^{N} \int_{0}^{L}\left(\left|\phi_{n}^{\prime}(x)\right|^{2}+\alpha^{2} \beta\left|\phi_{n}(x)\right|^{2}\right) d x, \\
& \int_{0}^{2 \pi}\left(\sum_{n=1}^{N}\left|\phi_{n}(x)\right|^{2}\right)^{3} d x \leq K_{2}(\beta)^{2} \sum_{n=1}^{N} \int_{0}^{2 \pi}\left(\left|\phi_{n}^{\prime}(x)\right|^{2}-\beta\left|\phi_{n}(x)\right|^{2}\right) d x
\end{aligned}
$$

and follow from interpolation inequalities (1.12), (1.13) by the method of [11].

Let us now consider two one-dimensional Schrödinger operators with periodic boundary conditions and matrix-valued potentials (1.14) and (1.15), where $\mathrm{P}$ is the orthogonal projection

$$
(\mathrm{P} \psi)(x)=\psi(x)-\frac{1}{2 \pi} \int_{0}^{2 \pi} \psi(t) d t
$$

acting component-wise.

Inequalities (3.2) and (3.3) in Theorem 3.1 are equivalent to the estimate of the 1-moments of the negative eigenvalues $-\lambda_{j} \leq 0$ and $-\mu_{j} \leq 0$ of the Schrödinger operators (1.14) and (1.15), respectively.

Theorem 3.2. Let $V$ be a non-negative $M \times M$ Hermitian matrix such that $\operatorname{Tr} V^{3 / 2} \in L_{1}$. Then the operators (1.14) and (1.15) have discrete spectrum, and their negative eigenvalues $\left\{-\lambda_{j}\right\}$ and $\left\{-\mu_{j}\right\}$ satisfy the estimates (1.16) and (1.17), respectively. 
Proof. Once we have the inequalities for the traces, the proof is completely analogous to the proof of Theorem 1 in [10] (see also Theorem 6.3 in [20].), and the assertion of the theorem is just the inequality $\mathrm{L}_{1,1} \leq \frac{2}{3 \sqrt{3}} \sqrt{\mathrm{k}_{1}}$ in the one dimensional matrix case, see (1.6). We include the proof of (1.17) for the sake of completeness (the proof of (1.16) is completely similar).

Let $\left\{\phi_{n}\right\}_{n=1}^{N}$ be the orthonormal eigen-vector functions corresponding to the negative eigenvalues $\left\{-\mu_{n}\right\}_{n=1}^{N}$ of (1.15) :

$$
-\phi_{n}^{\prime \prime}-\delta \phi_{n}-V \phi_{n}=-\mu_{n} \phi_{n}
$$

Using (3.3) and Hölder's inequality for traces we obtain

$$
\begin{aligned}
& \sum_{n=1}^{N} \mu_{n}= \\
& -\sum_{n=1}^{N} \sum_{j=1}^{M} \int_{0}^{2 \pi}\left(\left|\phi_{n}^{\prime}(x, j)\right|^{2}-\delta\left|\phi_{n}(x, j)\right|^{2}\right) d x+\int_{0}^{2 \pi} \operatorname{Tr}[V(x) U(x, x)] d x \leq \\
& \leq-K_{2}(\delta)^{-2} X+\left(\int_{0}^{2 \pi} \operatorname{Tr}\left[V(x)^{3 / 2}\right] d x\right)^{2 / 3} X^{1 / 3},
\end{aligned}
$$

where $X:=\int_{0}^{2 \pi} \operatorname{Tr}\left[U(x, x)^{3}\right] d x$. Calculating the maximum with respect to $X$ we obtain (1.17).

We observe that in terms of (1.3) the estimates (1.16) and (1.17) can be written in the form

$$
\begin{gathered}
\sum_{j} \lambda_{j} \leq \frac{\pi}{\sqrt{3}} K_{1}(\beta) \mathrm{L}_{1,1}^{\mathrm{cl}} \int_{0}^{L} \operatorname{Tr}\left[V(x)^{3 / 2}\right] d x \\
\sum_{j} \mu_{j} \leq \frac{\pi}{\sqrt{3}} K_{2}(\beta) \mathrm{L}_{1,1}^{\mathrm{cl}} \int_{0}^{2 \pi} \operatorname{Tr}\left[V(x)^{3 / 2}\right] d x .
\end{gathered}
$$

By using the Aizenmann-Lieb argument [1] we immediately obtain the following estimates for the Riesz means of order $\gamma \geq 1$ of the eigenvalues of the operators (1.14) and (1.15). 
Corollary 3.1. Let $V \geq 0$ be a $M \times M$ Hermitian matrix, such that $\operatorname{Tr} V^{\gamma+1 / 2} \in L_{1}$. Then for any $\gamma \geq 1$ the negative eigenvalues of the operators (1.14), (1.15) satisfy the inequalities

$$
\begin{gathered}
\sum_{j} \lambda_{j}^{\gamma} \leq \frac{\pi}{\sqrt{3}} K_{1}(\beta) \mathrm{L}_{\gamma, 1}^{\mathrm{cl}} \int_{0}^{L} \operatorname{Tr}\left[V(x)^{1 / 2+\gamma}\right] d x, \\
\sum_{j} \mu_{j}^{\gamma} \leq \frac{\pi}{\sqrt{3}} K_{2}(\beta) \mathrm{L}_{\gamma, 1}^{\mathrm{cl}} \int_{0}^{2 \pi} \operatorname{Tr}\left[V(x)^{1 / 2+\gamma}\right] d x .
\end{gathered}
$$

Proof. It is enough to prove this result for smooth matrix-valued potentials. Furthermore, we consider only (3.11), the treatment of the second operator being completely similar. Note that Theorem 3.2 is equivalent to

$$
\sum_{n} \lambda_{n} \leq \frac{2}{3 \sqrt{3}} K_{1}(\beta) \frac{1}{\mathrm{~L}_{1,1}^{\mathrm{cl}}} \int_{0}^{L} \int_{-\infty}^{\infty} \operatorname{Tr}\left[\left(|\xi|^{2}-V(x)\right)_{-}\right] \frac{d \xi d x}{2 \pi} .
$$

Scaling gives the simple identity for all $s \in \mathbb{R}$

$$
s_{-}^{\gamma}=C_{\gamma} \int_{0}^{\infty} t^{\gamma-2}(s+t)_{-} d t, \quad C_{\gamma}^{-1}=\mathcal{B}(\gamma-1,2),
$$

where $\mathcal{B}$ is the Beta function. Let $\left\{\nu_{j}(x)\right\}_{j=1}^{M}$ be the eigenvalues of the matrix-function $V(x)$. Then

$$
\begin{aligned}
& \sum_{n} \lambda_{n}^{\gamma}=C_{\gamma} \sum_{n} \int_{0}^{\infty} t^{\gamma-2}\left(-\lambda_{n}+t\right)_{-} d t \\
& \leq \frac{2 K_{1}(\beta)}{3 \sqrt{3}} \frac{C_{\gamma}}{\mathrm{L}_{1,1}^{\mathrm{cl}}} \int_{0}^{\infty} \int_{0}^{L} \int_{-\infty}^{\infty} t^{\gamma-2} \operatorname{Tr}\left[\left(|\xi|^{2}-V(x)+t\right)_{-}\right] \frac{d \xi d x}{2 \pi} d t \\
& =\frac{2 K_{1}(\beta)}{3 \sqrt{3}} \frac{C_{\gamma}}{\mathrm{L}_{1,1}^{\mathrm{cl}}} \sum_{j=1}^{M} \int_{0}^{\infty} \int_{0}^{L} \int_{-\infty}^{\infty} t^{\gamma-2} \operatorname{Tr}\left[\left(|\xi|^{2}-\nu_{j}(x)+t\right)_{-}\right] \frac{d \xi d x}{2 \pi} d t \\
& =\frac{2 K_{1}(\beta)}{3 \sqrt{3}} \frac{1}{\mathrm{~L}_{1,1}^{\mathrm{cl}}} \int_{0}^{L} \int_{-\infty}^{\infty} \operatorname{Tr}\left[\left(|\xi|^{2}-V(x)\right)_{-}^{\gamma}\right] \frac{d \xi d x}{2 \pi} \\
& =\frac{2 K_{1}(\beta)}{3 \sqrt{3}} \frac{\mathrm{L}_{\gamma, 1}^{\mathrm{cl}}}{\mathrm{L}_{1,1}^{\mathrm{cl}}} \int_{0}^{L} \operatorname{Tr}\left[V(x)^{1 / 2+\gamma}\right] d x
\end{aligned}
$$

which completes the proof since $\mathrm{L}_{1,1}^{\mathrm{cl}}=2 /(3 \pi)$.

\section{LIEB-THIRRING INEQUALITIES ON THE TORUS}

In this section we consider Lieb-Thirring inequalities on the torus (1.9), (1.10), paying special attention to the two-dimensional case. 
We recall the orthogonal projection $\mathrm{P}$ defined in (1.11), so that the resulting function has zero average with respect to shortest coordinate $x_{d}$ for all $x_{1}, \ldots, x_{d-1}$.

Theorem 4.1. Let $V \geq 0, \gamma \geq 1$, and let $V \in L_{\gamma+d / 2}\left(\mathbb{T}_{\alpha}^{d}\right)$. Then the negative eigenvalues $-\lambda_{n} \leq 0$ of the the Schrödinger operator

$$
\mathcal{H}=-\Delta-\mathrm{P}(V(x) \mathrm{P} \cdot)
$$

satisfy the bound

$$
\sum_{n} \lambda_{n}^{\gamma} \leq \mathrm{L}_{\gamma, d} \int_{\mathbb{T}_{\alpha}^{d}} V^{\gamma+d / 2}(x) d x
$$

where

$$
\mathrm{L}_{\gamma, d} \leq\left(\frac{\pi}{\sqrt{3}}\right)^{d} \prod_{j=1}^{d-1} K_{1}\left(\beta_{j}\right) K_{2}(\delta) \mathrm{L}_{\gamma, d}^{\mathrm{cl}},
$$

provided that $\beta_{j}>0, j=1, \ldots d-1$ are chosen so small that

$$
\delta:=\alpha_{1}^{2} \beta_{1}+\cdots+\alpha_{d-1}^{2} \beta_{d-1}<1 .
$$

If

$$
\beta_{j} \geq \beta_{*} \quad \& \quad \delta \leq \beta_{* *},
$$

(where the numbers $\beta_{*}$ and $\beta_{* *}$ are defined in Section 2), then

$$
\mathrm{L}_{\gamma, d} \leq\left(\frac{\pi}{\sqrt{3}}\right)^{d} \mathrm{~L}_{\gamma, d}^{\mathrm{cl}}
$$

For

$$
d \leq\left[\frac{\beta_{* *}}{\beta_{*}}\right]+1=19
$$

condition (4.4) can be satisfied for any $\alpha$, so that (4.5) always holds.

Proof. We write the operator $\mathcal{H}$ in the form

$$
\left(-\frac{d^{2}}{d x_{1}^{2}}+\alpha_{1}^{2} \beta_{1}\right)+\cdots+\left(-\frac{d^{2}}{d x_{d-1}^{2}}+\alpha_{d-1}^{2} \beta_{d-1}\right)+\left(-\frac{d^{2}}{d x_{d}^{2}}-\delta\right)-\mathrm{P}(V(x) \mathrm{P} \cdot) .
$$

We use the lifting argument with respect to dimensions developed in [24]. More precisely, we apply estimate (3.11) $d-1$ times with respect to variables $x_{1}, \ldots, x_{d-1}$, so that $\gamma$ is increased by $1 / 2$ at each step, and, finally, we use (3.12) with respect to $x_{d}$. Using the variational principle and denoting the negative pars of the operators by $[\cdot]_{-}$we obtain

$$
\sum_{n} \lambda_{n}^{\gamma}(\mathcal{H})=
$$




$$
\begin{aligned}
& =\sum_{n} \lambda_{n}^{\gamma}\left(-\partial_{1}^{2}-\alpha_{1}^{2} \beta_{1}+\sum_{j=2}^{d-1}\left(-\partial_{j}^{2}-\alpha_{1}^{2} \beta_{1}\right)+\left(-\partial_{d}^{2}+\delta-\mathrm{P}(V(x) \cdot)\right)\right) \leq \\
& \leq \sum_{n} \lambda_{n}^{\gamma}\left(-\partial_{1}^{2}-\alpha_{1}^{2} \beta_{1}+\right. \\
& \left.+\left[\sum_{j=2}^{d-1}\left(-\partial_{j}^{2}-\alpha_{1}^{2} \beta_{1}\right)+\left(-\partial_{d}^{2}+\delta-\mathrm{P}(V(x) \cdot)\right)\right]_{-}\right) \leq \\
& \leq \frac{\pi}{\sqrt{3}} K_{1}\left(\beta_{1}\right) \mathrm{L}_{\gamma, 1}^{\mathrm{cl}} \times \\
& \int_{0}^{L_{1}} \operatorname{Tr}\left[\sum_{j=2}^{d-1}\left(-\partial_{j}^{2}-\alpha_{1}^{2} \beta_{1}\right)+\left(-\partial_{d}^{2}+\delta-\mathrm{P}(V(x) \cdot)\right)\right]_{-}^{\gamma+1 / 2} d x_{1} \leq \\
& \text {........ } \\
& \leq\left(\frac{\pi}{\sqrt{3}}\right)^{d-1} \prod_{j=1}^{d-1}\left(K_{1}\left(\beta_{j}\right) \prod_{j=1}^{d-1} \mathrm{~L}_{\gamma+(j-1) / 2,1}^{\mathrm{cl}} \times\right. \\
& \int_{0}^{L_{1}} \cdots \int_{0}^{L_{d-1}} \operatorname{Tr}\left[\left(-\partial_{d}^{2}+\delta-\mathrm{P}(V(x) \cdot)\right)\right]_{-}^{\gamma+(d-1) / 2} d x_{1} \ldots d x_{d-1} \leq \\
& \leq\left(\frac{\pi}{\sqrt{3}}\right) \prod_{j=1}^{d} K_{1}\left(\beta_{j}\right) \prod_{j=1}^{d} \mathrm{~L}_{\gamma+(j-1) / 2,1}^{\mathrm{cl}} K_{2}(\delta) \int_{\mathbb{T}_{\alpha}^{d}} V^{\gamma+d / 2}(x) d x,
\end{aligned}
$$

which proves (4.2), (4.3) since (see (1.3))

$$
\prod_{j=1}^{d} \mathrm{~L}_{\gamma+(j-1) / 2,1}^{\mathrm{cl}}=\mathrm{L}_{\gamma, d}^{\mathrm{cl}}
$$

Finally, if we take $\beta_{j}=\beta_{*}=0.045 \ldots$, then $K_{1}\left(\beta_{j}\right)=1$. Since $\alpha_{j} \leq 1$, it follows that

$$
\delta=\sum_{j=1}^{d-1} \alpha_{j}^{2} \beta_{j}=\beta_{*} \sum_{j=1}^{d-1} \alpha_{j}^{2} \leq(d-1) \beta_{*} .
$$

Therefore the condition $\delta \leq \beta_{* *}=0.839 \ldots$ (giving $K_{2}(\delta)=1$ ) is always satisfied if

$$
d-1 \leq\left[\frac{\beta_{* *}}{\beta_{*}}\right]=18
$$

The proof is complete.

Remark 4.1. The complementary projection $\mathrm{Q}=I-\mathrm{P}$ maps $\dot{L}_{2}\left(\mathbb{T}_{\alpha}^{d}\right)$ onto $\dot{L}_{2}\left(\mathbb{T}_{\alpha^{\prime}}^{d-1}\right)$, where $\alpha^{\prime}=\left(\alpha_{1}, \ldots, \alpha_{d-1}\right)$ and $\dot{L}_{2}\left(\mathbb{T}_{\alpha^{\prime}}^{d-1}\right)$ is the subspace of $\dot{L}_{2}\left(\mathbb{T}_{\alpha}^{d}\right)$ of functions independent of $x_{d}$. 
Remark 4.2. If we compare (1.4) and (4.5), we see that the factor $\pi / \sqrt{3}$ accumulates in (4.5) at each iteration with respect to the dimension; while in (1.4) already at the second iteration sharp bounds [24] for the $\gamma$-moments with $\gamma \geq \frac{3}{2}$ are available, which are not known in the periodic case.

We single out the following corollary that is important for applications. Let $d=2$ so that $\mathbb{T}_{\alpha}^{2}=(0,2 \pi / \alpha) \times(0,2 \pi), \alpha \leq 1$.

Corollary 4.1. Let $V \geq 0$ and let $V \in L_{2}\left(\mathbb{T}_{\alpha}^{2}\right)$. Then the negative eigenvalues $-\lambda_{j} \leq 0$ of the operator (4.1) satisfy the following bound:

$$
\sum_{j} \lambda_{j} \leq\left(\frac{\pi}{\sqrt{3}}\right)^{2} \mathrm{~L}_{1,2}^{\mathrm{cl}} \int_{\mathbb{T}_{\alpha}^{2}} V^{2}(x) d x=\frac{\pi}{24} \int_{\mathbb{T}_{\alpha}^{2}} V^{2}(x) d x .
$$

Equivalently (see (1.5), (1.6)), if a family $\left\{\varphi_{j}\right\}_{j=1}^{N} \in \mathrm{P} H^{1}\left(\mathbb{T}_{\alpha}^{2}\right)$ is orthonormal, then $\rho_{\varphi}(x):=\sum_{j=1}^{N} \varphi_{j}(x)^{2}$ satisfies

$$
\int_{\mathbb{T}_{\alpha}^{2}} \rho_{\varphi}(x)^{2} d x \leq \frac{\pi}{6} \sum_{j=1}^{N}\left\|\nabla \varphi_{j}\right\|^{2}
$$

\section{Applications to attractors}

Starting from the important paper [26] the Lieb-Thirring inequalities play an essential role in the theory of attractors for infinite dimensional dissipative dynamical systems, especially, for the Navier-Stokes system. They are used for the first moments $(\gamma=1)$ in the equivalent formulation in terms of orthonormal families.

We first consider the square torus $\mathbb{T}^{2}=(0, L) \times(0, L)$.

Proposition 5.1. If $\left\{v_{j}\right\}_{j=1}^{m} \in \dot{H}^{1}\left(\mathbb{T}^{2}\right)$ is an orthonormal family of vector functions and $\operatorname{div} v_{j}=0$, then $\rho_{v}(x):=\sum_{j=1}^{m}\left|v_{j}(x)\right|^{2}$ satisfies

$$
\int_{\mathbb{T}^{2}} \rho_{v}(x)^{2} d x \leq c_{\mathrm{LT}} \sum_{j=1}^{m}\left\|\nabla v_{j}\right\|^{2}, \quad c_{\mathrm{LT}} \leq \frac{3}{2} .
$$

Proof. (See [19].) In the scalar case this follows from (1.7) and (1.5), (1.6). In two dimensions the passage to the vector case with $\operatorname{div} v_{j}=0$ does not increase the constant [18].

Turning to the applications we consider the damped and driven NavierStokes system

$$
\left\{\begin{array}{l}
\partial_{t} u+\sum_{i=1}^{2} u^{i} \partial_{i} u=\nu \Delta u-\mu u-\nabla p+g \\
\operatorname{div} u=0,\left.\quad u\right|_{t=0}=u_{0}
\end{array}\right.
$$


in a periodic square domain $\mathbb{T}^{2}=(0, L) \times(0, L)$. We assume that $g$ and $u$ have mean values zero. The system is studied in the small viscosity limit $\nu \rightarrow 0^{+}$, while the drag/damping coefficient $\mu>0$ is arbitrary but fixed.

Using the standard notation in the theory of the Navier-Stokes equations we denote by $H$ the closure in $L_{2}\left(\mathbb{T}^{2}\right)$ of the set of trigonometric polynomials with divergence and mean value zero. The norm $\|\cdot\|$ and scalar product $(\cdot, \cdot)$ in $H$ are those of $L_{2}\left(\mathbb{T}^{2}\right)$. We project the first equation onto $H$ and obtain the functional evolution equation

$$
\partial_{t} u+B(u, u)+\nu A u=-\mu u+g, \quad u(0)=u_{0},
$$

where $A$ is the Stokes operator and $B(u, v)$ is the nonlinear term defined as follows

$$
\langle A u, v\rangle=(\nabla u, \nabla v), \quad u, v \in H^{1} \cap H
$$

and

$$
\langle B(v, u), w\rangle=\int_{\mathbb{T}^{2}} \sum_{i, k=1}^{2} v^{k} \partial_{k} u^{i} w^{i} d x=: b(v, u, w),
$$

for all $u, v, w \in H^{1} \cap H$.

Equation (5.3) has a unique solution $u(t)$ and the solution semigroup $S_{t} u_{0} \rightarrow u(t)$ is well defined. The semigroup $S_{t}$ has a global attractor $\mathcal{A}$ which is a compact strictly invariant set in $H$ attracting under the action of $S_{t}$ all bounded sets as $t \rightarrow \infty$. These facts are well known for the classical Navier-Stokes equations [2], [9, 25], 31]; the case $\mu>0$ is similar. The solution semigroup $S_{t}$ is uniformly differentiable in $H$ with differential $L\left(t, u_{0}\right): \xi \rightarrow U(t) \in H$, where $U(t)$ is the solution of the variational equation

$\partial_{t} U=-\nu A U-\mu U-B(U, u(t))-B(u(t), U)=: \mathcal{L}\left(t, u_{0}\right) U, \quad U(0)=\xi$.

Furthermore, the differential $L\left(t, u_{0}\right)$ depends continuously on the initial point $u_{0} \in \mathcal{A}[2]$.

We estimate the numbers $q(m)$, that is, the sums of the first $m$ global Lyapunov exponents: [7], [9], 31]:

$$
q(m):=\limsup _{t \rightarrow \infty} \sup _{u_{0} \in \mathcal{A}} \sup _{\left\{v_{j}\right\}_{j=1}^{m} \in H \cap H^{1}} \frac{1}{t} \int_{0}^{t} \sum_{j=1}^{m}\left(\mathcal{L}\left(\tau, u_{0}\right) v_{j}, v_{j}\right) d \tau
$$

where $\left\{v_{j}\right\}_{j=1}^{m} \in H \cap H^{1}$ is an arbitrary orthonormal system of dimension $m$.

We first estimate the $H^{1}$-norm of the solutions on the attractor. Taking the scalar product of (5.3) with $A u$, using the identity $(B(u, u), A u)=0$ 
(see, for example, [9],[31]) and integrating by parts we obtain

$$
\partial_{t}\|\nabla u\|^{2}+2 \nu\|A u\|^{2}+2 \mu\|\nabla u\|^{2}=2(\nabla g, \nabla u) \leq \mu\|\nabla u\|^{2}+\mu^{-1}\|\nabla g\|^{2} .
$$

Dropping the $\nu$-term on the left-hand side, the Gronwall inequality gives

$$
\|\nabla u(t)\|^{2} \leq\|\nabla u(0)\|^{2} e^{-\mu t}+\frac{1-e^{-\mu t}}{\mu^{2}}\|\nabla g\|^{2},
$$

so that on the attractor $u(t) \in \mathcal{A}$ letting $t \rightarrow \infty$ we have a $\nu$-independent estimate

$$
\|\nabla u(t)\|^{2} \leq \frac{\|\nabla g\|^{2}}{\mu^{2}} .
$$

We now estimate the $m$-trace of the operator $\mathcal{L}$ in (5.6). Integrating by parts and using the identity $\left(B\left(u(t), v_{j}\right), v_{j}\right)=0$ (see [9], 31]) and the orthonormality of the $v_{j}$ 's, we obtain

$$
\sum_{j=1}^{m}\left(\mathcal{L}\left(t, u_{0}\right) v_{j}, v_{j}\right)=-\nu \sum_{j=1}^{m}\left\|\nabla v_{j}\right\|^{2}-\mu m-\sum_{j=1}^{m} b\left(v_{j}, u(t), v_{j}\right) .
$$

For the last term we use the point-wise inequality

$$
\left|\sum_{k, i=1}^{2} v^{k} \partial_{k} u^{i} v^{i}\right| \leq 2^{-1 / 2}|\nabla u||v|^{2}
$$

which holds for any $v=\left(v^{1}, v^{2}\right)$ and any $2 \times 2$ matrix $\nabla u=\left(\partial_{i} u^{j}\right)_{i, j=1}^{2}$ such that $\partial_{1} u^{1}+\partial_{2} u^{2}=0$, see [6, Lemma 4.1]. Using (5.9) and the Lieb-Thirring inequality (5.1) we obtain

$$
\begin{aligned}
& \sum_{j=1}^{m}\left(\mathcal{L}\left(t, u_{0}\right) v_{j}, v_{j}\right) \leq-\nu \sum_{j=1}^{m}\left\|\nabla v_{j}\right\|^{2}-\mu m+\frac{1}{\sqrt{2}} \int|\nabla u(t, x)| \rho_{v}(x) d x \leq \\
& -\nu \sum_{j=1}^{m}\left\|\nabla v_{j}\right\|^{2}-\mu m+\frac{1}{\sqrt{2}}\|\nabla u(t)\|\left\|\rho_{v}\right\| \leq \\
& -\nu \sum_{j=1}^{m}\left\|\nabla v_{j}\right\|^{2}-\mu m+\frac{1}{\sqrt{2}}\|\nabla u(t)\|\left(c_{\mathrm{LT}} \sum_{j=1}^{m}\left\|\nabla v_{j}\right\|^{2}\right)^{1 / 2} \leq \\
& -\nu \sum_{j=1}^{m}\left\|\nabla v_{j}\right\|^{2}-\mu m+\frac{c_{\mathrm{LT}}}{8 \nu}\|\nabla u(t)\|^{2}+\nu \sum_{j=1}^{m}\left\|\nabla v_{j}\right\|^{2}= \\
& -\mu m+\frac{c_{\mathrm{LT}}}{8 \nu}\|\nabla u(t)\|^{2} .
\end{aligned}
$$


Now we see from (5.6) and (5.7) that

$$
q(m) \leq-\mu m+\frac{c_{\mathrm{LT}}\|\nabla g\|^{2}}{8 \nu \mu^{2}} .
$$

We can proceed in a somewhat different way observing that

$$
m=\int \rho_{v}(x) d x \leq\left\|\rho_{v}\right\| L \quad \Rightarrow \quad \sum_{j=1}^{m}\left\|\nabla v_{j}\right\|^{2} \geq \frac{1}{c_{\mathrm{LT}}}\left\|\rho_{v}\right\|^{2} \geq \frac{1}{c_{\mathrm{LT}}} \frac{m^{2}}{L^{2}} .
$$

Then we argue as before but in the last but one line single out the term $\nu / 2 \sum_{j=1}^{m}\left\|\nabla v_{j}\right\|^{2}$ to absorb it in the half of the first term. We obtain

$$
q(m) \leq-\frac{\nu m^{2}}{2 c_{\mathrm{LT}} L^{2}}+\frac{c_{\mathrm{LT}}\|\nabla g\|^{2}}{4 \nu \mu^{2}} .
$$

Now if for an $m^{*}$ we have $q\left(m^{*}\right)<0$, then both the Hausdorff dimension [7, 31], and the fractal dimension [5, 6] of the attractor $\mathcal{A}$ satisfy

$$
\operatorname{dim}_{H} \mathcal{A} \leq \operatorname{dim}_{F} \mathcal{A}<m^{*} .
$$

Therefore estimates (5.10) and (5.11) along with (5.1) show that we have proved the following theorem.

Theorem 5.1. The fractal dimension of the attractor for the damped-driven Navier-Stokes system (5.2) satisfy the estimate

$$
\operatorname{dim}_{F} \mathcal{A} \leq \min \left(\frac{c_{\mathrm{LT}}}{8} \frac{\|\nabla g\|^{2}}{\nu \mu^{3}}, \frac{c_{\mathrm{LT}}}{\sqrt{2}} \frac{\|\nabla g\| L}{\nu \mu}\right) \leq \min \left(\frac{3}{16} \frac{\|\nabla g\|^{2}}{\nu \mu^{3}}, \frac{3}{2 \sqrt{2}} \frac{\|\nabla g\| L}{\nu \mu}\right) .
$$

Let us now consider the system (5.2) on the large elongated torus $\mathbb{T}_{\alpha}^{2}=$ $(0, L / \alpha) \times(0, L)$, where $\alpha \leq 1$. As before we assume that both scalar and vector functions have mean value zero over $\mathbb{T}_{\alpha}^{2}$, and we decompose the phase space $\dot{L}_{2}\left(\mathbb{T}_{\alpha}^{2}\right)=L_{2}\left(\mathbb{T}_{\alpha}^{2}\right) \cap \int_{\mathbb{T}_{\alpha}^{2}} u(x) d x=0$ into the orthogonal sum

$$
\dot{L}_{2}\left(\mathbb{T}_{\alpha}^{2}\right)=\mathrm{P} \dot{L}_{2}\left(\mathbb{T}_{\alpha}^{2}\right) \oplus \mathrm{Q} \dot{L}_{2}\left(\mathbb{T}_{\alpha}^{2}\right)
$$

where the orthogonal projection $\mathrm{P}$ is as in (1.11) in the $2 \mathrm{~d}$ case, and the projection Q

$$
(\mathrm{Q} \psi)\left(x_{1}\right)=\frac{1}{L} \int_{0}^{L} \psi\left(x_{1}, t\right) d t
$$

maps $\dot{L}_{2}\left(\mathbb{T}_{\alpha}^{2}\right)$ onto $\dot{L}_{2}(0, L / \alpha)$.

On the elongated torus we have the following Lieb-Thirring inequalities for orthonormal families. 
Proposition 5.2. If $\left\{v_{j}\right\}_{j=1}^{m} \in \mathrm{P} \dot{L}_{2}\left(\mathrm{~T}_{\alpha}^{2}\right)$ is an orthonormal family in $L_{2}\left(\mathrm{~T}_{\alpha}^{2}\right)$ and $\operatorname{div} v_{j}=0$, then $\rho_{\mathrm{P} v}(x):=\sum_{j=1}^{m}\left|v_{j}(x)\right|^{2}$ satisfies

$$
\int_{\mathrm{T}_{\alpha}^{2}} \rho_{\mathrm{P} v}(x)^{2} d x \leq c_{\mathrm{P}} \sum_{j=1}^{m}\left\|\nabla v_{j}\right\|^{2}, \quad c_{\mathrm{P}} \leq \frac{\pi}{6} .
$$

Accordingly, if $\left\{w_{j}\right\}_{j=1}^{m} \in \mathrm{Q} \dot{L}_{2}\left(\mathrm{~T}_{\alpha}^{2}\right)$ is an orthonormal family of vector functions and $\operatorname{div} w_{j}=0$, then $\rho_{\mathrm{Q} w}(x):=\sum_{j=1}^{m}\left|w_{j}(x)\right|^{2}$ satisfies

$$
\int_{\mathrm{T}_{\alpha}^{2}} \rho_{\mathrm{Q} w}(x)^{2} d x \leq \frac{c_{\mathrm{Q}}}{L} \sum_{j=1}^{m}\left\|\nabla w_{j}\right\|, \quad c_{\mathrm{Q}} \leq 6 .
$$

Proof. The proof of (5.14) is the same as in Proposition 5.1.

Turning to (5.15) we observe that $w_{j}$ depend only on $x_{1}$ and the family $\tilde{w}_{j}=\sqrt{L} w_{j}$ is orthonormal in $L_{2}(0, L / \alpha)$ with mean value zero. Then we use the one-dimensional Lieb-Thirring inequality for the operator of order one on $\dot{L}_{2}(0, L / \alpha)[16$

$$
\int_{0}^{L / \alpha}\left(\sum_{j=1}^{m} \tilde{\psi}(x)^{2}\right)^{2} d x \leq 6 \sum_{j=1}^{m}\left\|\tilde{\psi}_{j}^{(1 / 2)}\right\|_{L_{2}(0, L / \alpha)}^{2} \leq 6 \sum_{j=1}^{m}\left\|\tilde{\psi}_{j}^{\prime}\right\|_{L_{2}(0, L / \alpha)},
$$

where the second inequality follows from the interpolation inequality

$$
\left\|\psi^{(1 / 2)}\right\|^{2} \leq\|\psi\|\left\|\psi^{\prime}\right\|
$$

and orthonormality.

The conditions $w=\mathrm{Q} w=w\left(x_{1}\right)$ and $\operatorname{div} w=0$ imply that $\left(w^{1}\right)_{x_{1}}^{\prime}=0$ and hence $w^{1} \equiv 0$, therefore the inequality for vector functions is essentially a scalar inequality. Returning to $\psi_{j}$ and $w_{j}$ and to integrals over $\mathrm{T}_{\alpha}^{2}$ we obtain (5.15).

Finally, we observe that all the inequalities in the theorem also hold for suborthonormal families $\left\{v_{j}\right\}_{j=1}^{m}$, that is, satisfying

$$
\sum_{i, j=1}^{m} \xi_{i} \xi_{j}\left(v_{i}, v_{j}\right) \leq \sum_{i=1}^{m} \xi_{i}^{2} \quad \text { for every } \xi \in \mathbb{R}^{m}
$$

and the corresponding constants do not increase [18].

We are now prepared to state the main result of this section in which we estimate the fractal dimension of the attractor $\mathcal{A}$ of system (5.2) on the torus $\mathbb{T}_{\alpha}^{2}$ paying special attention to the dependence of the estimates on $\alpha \rightarrow 0^{+}$and $\nu \rightarrow 0^{+}$. 
Theorem 5.2. The damped Navier-Stokes system (5.2) on the elongated torus $\mathrm{T}_{\alpha}^{2}$ has the attractor $\mathcal{A}$ and

$$
\operatorname{dim}_{F} \mathcal{A} \leq\left(\frac{c_{\mathrm{P}}}{2}+\sqrt{c_{\mathrm{P}} c_{\mathrm{Q}}}\right) \frac{\|\nabla g\|^{2}}{\nu \mu^{2}} \leq\left(\frac{\pi}{12}+\sqrt{\pi}\right) \frac{\|\nabla g\|^{2}}{\nu \mu^{3}}
$$

for all sufficiently small $\nu \leq 8 \mu L^{2}$.

Proof. As before we have (5.8)

$$
\sum_{j=1}^{m}\left(\mathcal{L}\left(t, u_{0}\right) v_{j}, v_{j}\right)=-\nu \sum_{j=1}^{m}\left\|\nabla v_{j}\right\|^{2}-\mu m-\sum_{j=1}^{m} b\left(v_{j}, u(t), v_{j}\right),
$$

and the main task is to estimate the last term there. The main idea [33] is decompose the solution $u$ and the $v_{j}$ 's as follows

$$
u=\mathrm{P} u+\mathrm{Q} u, \quad v_{j}=\mathrm{P} v_{j}+\mathrm{Q} v_{j}
$$

and use $\alpha$-independent Lieb-Thirring inequalities in Theorem 5.2. We note that since the $v_{j}$ 's are orthonormal, both $\mathrm{P} v_{j}$ and $\mathrm{Q} v_{j}$ are suborthonormal.

Since $\partial_{1} \mathrm{Q}=\mathrm{Q} \partial_{1}$ and $\partial_{2} \mathrm{Q}=\mathrm{Q} \partial_{2}=0$, it follows that $\operatorname{div} \mathrm{P} w=\operatorname{div} \mathrm{Q} w=$ 0 if $\operatorname{div} w=0$. Since $\mathrm{Q} u$ and $\mathrm{Q} v_{j}$ depend only on $x_{1}, \mathrm{Q} u^{1}=\mathrm{Q} v_{j}^{1}=0$ and $\int_{0}^{L} \mathrm{P} u\left(x_{1}, x_{2}\right) d x_{2}=0$, it follows that

$$
b\left(\mathrm{Q} v_{j}, u, \mathrm{Q} v_{j}\right)=0, \quad b\left(\mathrm{Q} v_{j}, \mathrm{Q} u, \mathrm{P} v_{j}\right)=0, \quad b\left(\mathrm{P} v_{j}, \mathrm{Q} u, \mathrm{Q} v_{j}\right)=0 .
$$

For example,

$$
b(\mathrm{Q} v, u, \mathrm{Q} v)=\int_{0}^{L / \alpha}\left(\mathrm{Q} v^{2}\left(x_{1}\right)\right)^{2} d x_{1} \int_{0}^{L} \partial_{2} u^{2}\left(x_{1}, x_{2}\right) d x_{2}=0
$$

by periodicity. Therefore

$$
b\left(v_{j}, u, v_{j}\right)=b\left(\mathrm{P} v_{j}, u, \mathrm{P} v_{j}\right)+b\left(\mathrm{Q} v_{j}, \mathrm{P} u, \mathrm{P} v_{j}\right)+b\left(\mathrm{P} v_{j}, \mathrm{P} u, \mathrm{Q} v_{j}\right) .
$$

Hence, in view of (5.9)

$$
\sum_{j=1}^{m} b\left(v_{j}, u, v_{j}\right) \leq \frac{1}{\sqrt{2}}\|\nabla u\|\left\|\rho_{\mathrm{P} v}\right\|+\sqrt{2}\|\nabla \mathrm{P} u\|\left\|\rho_{\mathrm{Q} v}\right\|^{1 / 2}\left\|\rho_{\mathrm{P} v}\right\|^{1 / 2},
$$

For the first term we use (15.14) and single out $\nu / 2 \sum_{j=1}^{m}\left\|\nabla \mathrm{P} v_{j}\right\|^{2}$ :

$$
\frac{1}{\sqrt{2}}\|\nabla u\|\left\|\rho_{\mathrm{P} v}\right\| \leq \frac{c_{\mathrm{P}}}{4 \nu}\|\nabla u\|^{2}+\frac{\nu}{2} \sum_{j=1}^{m}\left\|\nabla \mathrm{P} v_{j}\right\|^{2}
$$


For the second term we use (5.14), estimate (5.15) in the form

$$
\left\|\rho_{\mathrm{Q} v}\right\|^{2} \leq \frac{c_{\mathrm{Q}}}{L} \sqrt{m}\left(\sum_{j=1}^{m}\left\|\nabla w_{j}\right\|^{2}\right)^{1 / 2}
$$

and single out the terms $\nu / 2 \sum_{j=1}^{m}\left\|\nabla \mathrm{P} v_{j}\right\|^{2}$ and $\nu \sum_{j=1}^{m}\left\|\nabla \mathrm{Q} v_{j}\right\|^{2}$ :

$$
\begin{aligned}
& \sqrt{2}\|\nabla \mathrm{P} u\|\left\|\rho_{\mathrm{Q} v}\right\|^{1 / 2}\left\|\rho_{\mathrm{P} v}\right\|^{1 / 2} \leq \\
& \sqrt{2}\|\nabla u\|\left(c_{\mathrm{P}} c_{\mathrm{Q}}\right)^{1 / 4}\left(\sum_{j=1}^{m}\left\|\nabla \mathrm{P} v_{j}\right\|^{2}\right)^{1 / 4}\left(\frac{m}{L^{2}} \sum_{j=1}^{m}\left\|\nabla \mathrm{Q} v_{j}\right\|^{2}\right)^{1 / 8} \leq \\
& \frac{\|\nabla u\|^{2} \sqrt{c_{\mathrm{P}} c_{\mathrm{Q}}}}{2 \nu}+\nu\left(\sum_{j=1}^{m}\left\|\nabla \mathrm{P} v_{j}\right\|^{2}\right)^{1 / 2}\left(\frac{m}{L^{2}} \sum_{j=1}^{m}\left\|\nabla \mathrm{Q} v_{j}\right\|^{2}\right)^{1 / 4} \leq \\
& \frac{\|\nabla u\|^{2} \sqrt{c_{\mathrm{P}} c_{\mathrm{Q}}}}{2 \nu}+\frac{\nu}{2} \sum_{j=1}^{m}\left\|\nabla \mathrm{P} v_{j}\right\|^{2}+\nu \sum_{j=1}^{m}\left\|\nabla \mathrm{Q} v_{j}\right\|^{2}+\frac{\nu m}{16 L^{2}} \text {. }
\end{aligned}
$$

Since $\|\nabla v\|^{2}=\|\mathrm{P} \nabla v\|^{2}+\|\mathrm{Q} \nabla v\|^{2}$, we obtain

$$
\sum_{j=1}^{m} b\left(v_{j}, u(t), v_{j}\right) \leq \frac{1}{2 \nu}\left(\frac{c_{\mathrm{P}}}{2}+\sqrt{c_{\mathrm{P}} c_{\mathrm{Q}}}\right)\|\nabla u(t)\|^{2}+\nu \sum_{j=1}^{m}\left\|\nabla v_{j}\right\|^{2}+\frac{\nu m}{16 L^{2}} .
$$

Substituting this into (5.17) and using (5.7) we finally obtain the estimate for the sum of $m$ global Lyapunov exponents:

$$
q(m) \leq \frac{1}{2}\left(\frac{c_{\mathrm{P}}}{2}+\sqrt{c_{\mathrm{P}} c_{\mathrm{Q}}}\right) \frac{\|\nabla g\|^{2}}{\nu \mu^{2}}-\mu m\left(1-\frac{\nu}{16 \mu L^{2}}\right) .
$$

If $\nu \leq 8 \mu L^{2}$, then for

$$
m^{*}=\left(\frac{c_{\mathrm{P}}}{2}+\sqrt{c_{\mathrm{P}} c_{\mathrm{Q}}}\right) \frac{\|\nabla g\|^{2}}{\nu \mu^{3}}
$$

$q\left(m^{*}\right) \leq 0$, and hence

$$
\operatorname{dim}_{F} \mathcal{A} \leq m^{*}
$$

The proof is complete.

We finally point out that estimate (5.12) for the square torus is sharp as $\nu \rightarrow 0^{+}$and estimate (5.16) is sharp as both $\nu \rightarrow 0^{+}$and $\alpha \rightarrow 0^{+}$. The lower bounds for the dimension of the attractor are based on the characterization of the attractor as the section at any given time of the set of all complete trajectories bounded for $t \in \mathbb{R}$. Therefore all stationary solutions and their unstable manifolds lie on the attractor. Such an unstable stationary solution 
for the 2d periodic Navier-Stokes was first constructed in [29] and is called the Kolmogorov flow. The construction was generalized in [28] to prove that the estimate for the dimension obtained in 8] is logarithmically sharp. It also applies to our periodic damped Navier-Stokes system [21, 22]. In particular, it was shown in [22] that the right-hand side

$$
g=g_{s}=\left\{\begin{array}{l}
g_{1}=c_{1} \nu^{2} s^{3} \sin s x_{2}, \\
g_{2}=0,
\end{array}\right.
$$

where $c_{1}$ is an absolute constant and $\mathbb{T}_{\alpha}^{2}=(0,2 \pi / \alpha) \times(0,2 \pi)$, produces the stationary solution with unstable manifold of dimension

$$
d=c_{2} \frac{s^{2}}{\alpha}
$$

where $s \gg 1$. Setting $s:=\sqrt{\frac{\mu}{\nu}}$ we find that $\operatorname{dim} \mathcal{A} \geq d=c_{2} \frac{\mu}{\alpha \nu}$. Since $g$ is independent of $x_{1}$, it follows that

$$
\left\|\nabla g_{s}\right\|^{2}=c_{3} \frac{\nu^{4} s^{8}}{\alpha}=c_{3} \frac{\mu^{4}}{\alpha}
$$

so that for $g=g_{s}$ the dimensionless number $\frac{\|\nabla g\|^{2}}{\nu \mu^{3}}$ becomes

$$
\frac{\|\nabla g\|^{2}}{\nu \mu^{3}}=c_{3} \frac{\mu}{\alpha \nu}
$$

and the upper bound in (5.16) for $\mathcal{A}=\mathcal{A}_{s}$ is supplemented with a sharp lower bound

$$
\operatorname{dim} \mathcal{A}_{s} \geq \frac{c_{2}}{c_{3}} \frac{\|\nabla g\|^{2}}{\nu \mu^{3}},
$$

where $c_{2}$ and $c_{3}$ are some absolute constants (which can be calculated).

Acknowledgements. A.I. acknowledges financial support from the Russian Science Foundation (grant no. 14-21-00025).

\section{REFERENCES}

[1] M. Aizenman and E.H. Lieb, On semi-classical bounds for eigenvalues of Schrödinger operators. Phys. Lett. 66A (1978), 427-429.

[2] A.V. Babin A.V and M.I. Vishik, Attractors of Evolution Equations. Nauka, Moscow, 1989; English transl. North-Holland, Amsterdam, 1992.

[3] M.V. Bartuccelli, J. Deane, and S.V.Zelik, Asymptotic expansions and extremals for the critical Sobolev and Gagliardo-Nirenberg inequalities on a torus. Proc. Roy. Soc. Edinburgh 143A (2013), 445-482.

[4] R. Benguria and M. Loss, A simple proof of a theorem by Laptev and Weidl. Math. Res. Lett. 7 (2000), 195-203. 
[5] V. Chepyzhov and A. Ilyin, A note on the fractal dimension of attractors of dissipative dynamical systems. Nonlinear Anal. Theory, Methods $\&$ Applications, 44 (2001), 811-819.

[6] V.V. Chepyzhov and A.A. Ilyin, On the fractal dimension of invariant sets; applications to Navier-Stokes equations. Discrete and Continuous Dynamical Systems 10, nos.1\&2 (2004) 117-135.

[7] P. Constantin and C. Foias, Global Lyapunov exponents, Kaplan-Yorke formulas and the dimension of the attractors for the 2D Navier-Stokes equations. Comm. Pure Appl. Math. 38 (1985), 1-27.

[8] P. Constantin, C. Foias, and R. Temam, On the dimension of the attractors in twodimensional turbulence. Physica D 30 (1988), 284-296.

[9] P. Constantin and C. Foias, Navier-Stokes Equations. The University of Chicago Press, 1988.

[10] J. Dolbeault, A. Laptev, and M. Loss, Lieb-Thirring inequalities with improved constants. J. European Math. Soc. 10:4 (2008), 1121-1126.

[11] A. Eden and C. Foias. A simple proof of the generalized Lieb-Thirring inequalities in one space dimension. J. Math. Anal. Appl. 162 (1991), 250-254.

[12] J.M. Ghidaglia, M. Marion, and R. Temam, Generalization of the Sobolev-LiebThirring inequalities and applications to the dimension of attractors. Differential and Integral Equations 1:1 (1988), 1-21.

[13] J.M. Ghidaglia and R. Temam, Lower bound on the dimension of the attractor for the Navier-Stokes equations in space dimension 3. Mechanics, analysis and geometry: 200 years after Lagrange, 33-60, North-Holland, Amsterdam, 1991.

[14] G.H. Hardy, A note on two inequalities. J. London Math. Soc. 11 (1936), 167-170.

[15] D. Hundertmark, A. Laptev, and T. Weidl, New bounds on the Lieb-Thirring constants. Inventiones Mathematicae, 140:3 (2000), 693-704.

[16] A.A. Ilyin, Lieb-Thirring inequalities on the $N$-sphere and in the plane, and some applications. Proc. London Math. Soc. 67, 159-182 (1993).

[17] A.A. Ilyin, Best constants in multiplicative inequalities for sup-norms. J. London Math. Soc.(2) 58, 84-96 (1998).

[18] A.A. Ilyin, Lieb-Thirring integral inequalities and their applications to attractors of the Navier-Stokes equations. Mat. Sbornik 196:1, 33-66 (2005); English transl. in Sb. Math. 196:1 (2005).

[19] A.A. Ilyin, Lieb-Thirring inequalities on some manifolds. J. Spectr. Theory 2, 57-78 (2012).

[20] A. Ilyin, A. Laptev, M. Loss, and S.Zelik, One-dimensional interpolation inequalities, Carlson-Landau inequalities and magnetic Schrödinger operators. Int. Math. Res. Not. (2015), doi:10.1093/imrn/rnv156.

[21] A.A. Ilyin, A. Miranville, and E.S. Titi, A small viscosity sharp estimate for the global attractor of the 2-D damped-driven Navier-Stokes equations. Commun. Math. Sciences 2, no. 3 (2004), 403-426.

[22] A.A. Ilyin and E.S. Titi, The damped-driven 2D Navier-Stokes system on large elongated domains. J. Math. Fluid Mech. 10 (2008), 159-175.

[23] B.S. Kashin, On a class of inequalities for orthonormal systems. Mat. Zametki 80:2 (2006), 204-208; English transl. Math. Notes 80 (2006), 199-203. 
[24] A. Laptev and T. Weidl, Sharp Lieb-Thirring inequalities in high dimensions. Acta Math. 184 (2000), 87-111.

[25] O.A. Ladyzhenskaya, Attractors for Semigroups and Evolution Equations. Leizioni Lincei. Cambridge Univ. Press, Cambridge, 1991.

[26] E.H. Lieb, On characteristic exponents in turbulence. Comm. Math. Phys. 92 (1984), 473-480.

[27] E. Lieb and W. Thirring, Inequalities for the moments of the eigenvalues of the Schrödinger Hamiltonian and their relation to Sobolev inequalities, Studies in Mathematical Physics. Essays in honor of Valentine Bargmann, Princeton University Press, Princeton NJ, 269-303 (1976).

[28] V.X. Liu, A sharp lower bound for the Hausdorff dimension of the global attractors of the 2D Navier-Stokes equations. Comm. Math. Phys. 158 (1993), 327-339.

[29] L.D. Meshalkin and Ya.G. Sinai, Investigation of the stability of a stationary solution of a system of equations for the plane movement of an incompressible viscous liquid. Priklad. Mat. Mekh.. 25, 1140-1143 (1961), English transl. in J. Appl. Math. Mech. 25 (1961).

[30] L.V. Taikov, Kolmogorov-type inequalities and the best formulas for numerical differentiation. Mat. Zametki 4, 233-238 (1968); English transl. Math. Notes 4 (1968), 631-634.

[31] R. Temam, Infinite Dimensional Dynamical Systems in Mechanics and Physics, 2nd Edition. New York, Springer-Verlag, 1997.

[32] S.V.Zelik and A.A. Ilyin, Green's function asymptotics and sharp interpolation inequalities. Uspekhi Mat. Nauk 69:2 (2014), 23-76; English transl. in Russian Math. Surveys 69:2 (2014).

[33] M. Ziane, Optimal bounds on the dimension of attractors for the Navier-Stokes equations. Physica D 105, 1-19 (1997).

Keldysh Institute of Applied Mathematics;

ImPerial College London And Institute MitTaG-Leffler;

E-mail address: ilyin@keldysh.ru; a.laptev@imperial.ac.uk 